\section{IDENTIDAD DE GÉNERO: MODELOS EXPLICATIVOS}

\author{
Patricia García-Leiva \\ Departamento de Psicología Area de Psicología Social \\ Universidad de Huelva
}

e-mail: patriciag11973@yahoo.es
GENDER IDENTITY: MODELS
Resumen El presente artículo lleva a cabo una revisión teórica de modelos que han tratado de explicar el self de género como un fenómeno social. Se recorren teorías de marcado acento cognitivo y se tratan los modelos sociocognitivos para llegar a los modelos sociales. La explicación aportada por la Teoría de la Identidad Social (Tajfel, 1981 y Tajfel y Turner, 1986) y la reciente propuesta integradora entre la Psicología y la Sociología de Deaux y Martin (2003) constituyen el hilo conductor de la revisión.
Abstract This article is a self identity theory review developed in a framework from socials models. It describes cognitive models, cognitive-social models and social models. The Tajfel's Social Identity Theory (Tajfel, 1981 y Tajfel y Turner, 1986) and the Deaux y Martin's new model that integrates Psychological and Sociological traditions are the main arguments along the paper.
Palabras clave Identidad, género, teorías de la identidad, Modelos sociales.
Key words Identity, gender, Identity Theories, Socials models. 


\section{INTRODUCCIÓN}

Tradicionalmente el estudio del self de género se ha entendido como el análisis de su contenido, de sus fases de adquisición y de los procesos intrapsíquicos responsables de la identificación. Pero la Psicología Social se ha interesado en evaluar cómo el entorno influye en el desarrollo del self. Por ello ha querido saber qué papel juegan las dinámicas grupales, las estructuras sociales y cómo interaccionan con los elementos cognitivos y motivacionales. Este va a ser el objetivo de este artículo: examinar el proceso de construcción del self de género de una persona en relación con los roles y estereotipos dominantes en una estructura social.

Con esta finalidad se va a realizar una revisión cualitativa y crítica de teorías que han tratado de dar respuesta a tan complejo fenómeno. Se comienza con los modelos cognitivos, se tratan los sociocognitivos y se finaliza con los sociales. De todos ellos se presta especial interés a las denominadas teorías de la identidad social por ser perspectivas integradoras que escapan a los primeros análisis reduccionistas. En ellas se recogen los factores intrasujeto y su interacción con los elementos situacionales, los procesos de aprendizaje y las presiones sociales y estructurales. Gracias a este análisis global pueden explicar la construcción, modificación y mantenimiento de la identidad de género.

De las tres teorías de la identidad social la planteada por Tajfel y Turner (SIT, 1981) y la propuesta de Deaux y Martin (2003) van a ser las protagonistas: la primera por su amplio bagaje teórico y empírico, la segunda por su innovación e integración interdisciplinar. Ambas explican la identidad de género como un fenómeno social dinámico y multicausal, pero mientas la SIT peca de psicologicista, la segunda carece de apoyo empírico, limitaciones que en ambos casos son subsanables. Especialmente en la propuesta de Deaux y Martin, pues estas autoras unen la teoría sociológica de la identidad (IT de Stryker, 1980; Stryker y Serpe, 1982) a la SIT para evitar su déficit de análisis social.

Con esta revisión se quiere contribuir a resaltar la importancia de la unión interdisciplinar apoyando así la iniciativa de Deaux y Martin (2003), una iniciativa que es necesaria en objetos de estudios tan complejos como son los seres humanos.

Pero antes de comenzar el recorrido teórico es necesario acercarse a la concepción de género y su relación con su acompañante biológico, el sexo.

\section{DEL SEXO AL GÉNERO}

La concepción del género como lo social, lo cultural y el sexo como la división biológica hombre-mujer es la propuesta dominante en la actualidad. Este planteamiento defiende la existencia de una continua interacción entre lo biológico y lo cultural, entre el dimorfismo sexual, hombre-mujer, y el producto sociocultural de cada uno de ellos: masculinidad-feminidad. La propuesta se amplía posteriormente a cuatro categorías con la inclusión de andróginos e indiferenciados (Spence, Helmreich y Stapp, 1974).

En definitiva se construye lo que cada estructura social cree y requiere para cada morfismo sexual. "El género se puede entender como una creación simbólica que pone en cuestión el dictum esencialista de la biología es destino, trascendiendo dicho reduccionismo, al interpretar las relaciones entre varones y mujeres como construcciones culturales, que derivan de imponer significados sociales, culturales y psicológicos al dimorfismo sexual aparente" (Bonilla, 1998, p. 149). Posee, por tanto, un desarrollo dinámico en el que la persona es parte activa en la continua retroalimentación entre la biología y el medio sin que se puedan separar.

Tres son las corrientes teóricas que han tratado de explicar el origen del género: la sociobiológica, el constructivismo social y la psicodinámica. Las tres consideran que las diferencias de género son estables en el tiempo pero difieren en el origen de estas diferencias.

La primera, la sociobiología, plantea un origen biológico y trata de explicar el comportamiento de género en base a las distintas estrategias adoptadas por varones y mujeres, a lo largo de la evolución, para garantizar el éxito reproductivo de la especie.

La segunda busca los orígenes de las diferencias de género en el ámbito socio-cultural. De acuerdo con este planteamiento el género es una construcción del lenguaje, la historia y la cultura concreta (Hare-Mustin y Marecek, 1994) en un tiempo y en un lugar específico. Por tanto, dentro de un mismo contexto cultural pueden coexistir distintas construcciones de género (Barberá, 1998).

Y la tercera propone que la génesis del género reside en el proceso de identificación primaria. Mientras el niño tiene su objeto de deseo en la madre y se identifica con el padre, la niña tiene su objeto de deseo en el padre y se identifica con la madre.

De las tres líneas teóricas las dos primeras son las que gozan de mejor salud en la actualidad. El constructivismo social es uno de los modelos más referenciados por los/as teóricos/as feministas, aunque la sociobiología resurge con fuerza en los últimos años. 


\section{LA IDENTIDAD DE GÉNERO: CONCEPTO Y MODELOS} TEÓRICOS

Cuando nacemos somos clasificados en dos grandes grupos: niños y niñas. Cuando crecemos, al mismo tiempo que desarrollamos la conciencia de ser un individuo distinto de los demás, el denominado self existencial, adquirimos el self sexual, la autocategorización como hombre o como mujer, y junto con éste el self de género. La división biológica que traemos conlleva diferencias reproductivas pero no diferencias actitudinales, normativas, conductuales o de roles. Todo ello es producto de la asignación social. La identidad de género es la autoclasificación como hombre o mujer sobre la base de lo que culturalmente se entiende por hombre o mujer (López, 1988a). Es el conjunto de sentimientos y pensamientos que tiene una persona en cuanto miembro de una categoría de género (Carver, Yunger y Perry, 2003).

El proceso de construcción del self de género acontece a nivel intraindiviual pero se desarrolla en interacción con el aprendizaje de roles, estereotipos y conductas (Barberá, 1998). Esto no implica la asunción de los roles y actitudes de género, puesto que cada persona desarrolla su propio sentido de masculinidad y feminidad (Spence, 1993; Koestner y Aube, 1995).

Sobre cómo se produce dicha construcción e interacción hay múltiples explicaciones. Tradicionalmente se han adoptado dos líneas argumentales: 1) aquella que se centra en los procesos internos al sujeto y 2) la que estudia la interacción entre los procesos psicológicos básicos y los factores sociales y situacionales. Dentro del primer grupo se encuentran las teorías cognitivas. Los modelos de interacción sociocognitiva y los denominados modelos sociales, siguiendo a Barberá (1998), se expondrán dentro del segundo grupo.

\section{Teorías Cognitivas}

Aquí estarían representadas dos líneas de trabajo: la basada en las Teorías Genético-Evolutivas (Piaget, 1966 y Kohlberg, 1981) y las basadas en el concepto de esquema (Bem, 1981; Markus y Oyserman, 1989). Para ambas el elemento central en la construcción de la identidad de género es la autocategorización (López, 1988a). Igualmente comparten el énfasis en la estabilidad del constructo género y el papel protagonista de la persona en el proceso identitario.

\subsection{Koblberg y J. Piaget}

Desde la psicología cognitiva se ha defendido la existencia de tres etapas en el desarrollo de la propia asigna- ción grupal. De acuerdo con Kohlberg (1966), y siguiendo los estadios de Piaget (1966), son: el etiquetaje, la estabilidad y la constancia de género. La primera etapa consiste en la categorización de uno mismo y de los demás en dos grupos diferentes, hombres y mujeres. Posteriormente el niño comprende que esa categoría a la que pertenece se mantiene a pesar de los cambios superficiales, como ropas o adornos. Por último se asimila la constancia de género a pesar de la variabilidad situacional. Kohlberg considera que estos estadios acontecen a los dos años el primero, entre los dos y los cinco el segundo y a partir de cinco el tercero, aunque dicha cronología parece ser variable (Slaby y Frey, 1975).

Entre las críticas al modelo se encuentra su acento en la primera infancia obviando el desarrollo y cambios de la identidad de género a lo largo del ciclo vital. Un ejemplo de estos cambios es la tendencia a la androginia en la edad adulta (Lòpez, 1988b).

Otra de las críticas que se le ha realizado alude a la secuencia. Kohlberg considera que la adquisición de la identidad de género es previa a la manifestación de comportamientos tipificados y de estereotipos de género. Sin embargo, son diversas las investigaciones que recogen comportamientos tipificados y manifestaciones estereotípicas previas al self de género (Golombok y Fivush, 1994).

Por último, plantea una causalidad lineal directa de los estadios genéticos evolutivos: factor partícipe, pero no responsable único en la adquisición de la identidad de género, como exponen otras teorías.

\subsection{S. Bem y H. Markus}

El esquema, concepto desarrollado por la corriente del procesamiento de la información, sería la estructura que orienta y organiza las percepciones de las personas (Huston, 1983). Una vez que alguien se autocategoriza como hombre o como mujer, procesa e interpreta la información en base a su pertenencia grupal, siendo el contexto el que determina qué es lo adecuado para cada género (Martin y Halverson, 1983).

El esquema supone una estructura que indica roles, estereotipos, conductas, rasgos de personalidad, etcétera (Bem, 1981). Se convierte en el andamio sobre el que se construyen nuestras cogniciones, nuestras evaluaciones y nuestros comportamientos. Desde el momento en que la persona se autoclasifica como miembro de un grupo su interpretación del mundo y de sí mismo va a seguir las directrices del mismo. Puesto que el esquema se aplica de forma automática (Biernat, Manis y Nelson, 1991) es difícil modificarlo, pero a pesar de ello presen- 
ta una alta variabilidad interindividual (Markus y cols. 1982; Silka y Maslach, 1987).

Las diferencias entre Bem y Markus provienen de cómo influye el género en el concepto de la persona. Para Markus el autoconcepto de género es el elemento que organiza nuestras vivencias (Markus y cols, 1982); sin embargo, para Bem (1981) el autoconcepto está supeditado al esquema de género. Además mientras Markus habla de autoconcepto masculino, femenino, ambos o ninguno, Bem discrimina entre personas esquemáticas o no esquemáticas. De acuerdo con esta autora la persona esquematiza interpreta la realidad siguiendo fielmente los constructos de masculinidad y feminidad. Sin embargo, la no esquemática o andrógina, aunque conoce los roles y estereotipos dominantes, actúa e interpreta su entorno con independencia de ellos.

Esta propuesta ha tenido un gran impacto, entre otros factores, por el extenso uso del cuestionario elaborado por Bem para la medición de la identidad de género, el BSRI (1974). Pero también presenta limitaciones. De ellas se puede destacar su incapacidad para explicar qué hace que las personas se identifiquen con mayor o menor intensidad o qué papel juegan las estructuras sociales y las dinámicas grupales en la identidad de género. Son limitaciones que habría que unir a las diversas críticas que ha recibido el concepto de esquema. Los modelos que siguen tratan de subsanar estas deficiencias.

\section{Perspectiva social}

Tal y como se ha comentado al inicio de esta revisión teórica, este apartado incluye los Modelos de Interacción Sociocognitiva y los Modelos Sociales (Barberá, 1998). Ambos se exponen juntos pues explican el proceso de construcción de género desde la interacción del contexto y los procesos intrasujeto.

\subsection{Modelos de interacción sociocognitiva}

Esta línea de trabajo se basa en la hipótesis de la profecía autocumplida (Merton, 1948). Se toma como referencia puesto que en ella están presentes todos los procesos que explican el comportamiento humano: cognitivos, motivacionales, comportamentales, estructura social y la situación específica (Barberá, 1998). Cuando dos personas interactúan los elementos intrasujetos de ambos y los sociales entran en interacción. Esta interacción da lugar a una construcción diferente en función del rol que tiene cada una de las personas: el que perci- be, el que actúa o ambos. El género sería por tanto una construcción-acción.

Aunque es un proceso que se retroalimenta permanentemente parece empezar con los estereotipos. Éstos, que han sido adquirimos a lo largo del proceso de socialización, condicionan la forma en la que se procesa la información. Por tanto influyen en la interacción y la construcción de la realidad. Sus diversos mecanismos de mantenimiento, como el sesgo confirmatorio (Snyder, 1981; Stangor y Ford, 1992), los perpetúan y dificultan su modificación.

En segundo lugar los esquemas estereotípicos condicionan las actitudes y acciones reforzando las creencias de partida. El "efecto Rosenthal" (Rosenthal y Jacobson, 1968) muestra cómo los individuos no se limitan a prestar atención sesgada a la información para reforzar sus creencias, sino que además son capaces de generar las condiciones necesarias para obtener la confirmación de las mismas (Darley y Fazio, 1980; Snyder, 1981).

En tercer lugar los comportamientos cierran el ciclo de autocumplimiento. Los procesos de atribución causales propician el reforzamiento de los estereotipos ya que aquellas conductas que no cumplen lo esperado se interpretan como excepciones. De igual forma el error fundamental de atribución facilita que las construcciones de lo masculino y lo femenino se interpreten como propias del grupo de hombres y mujeres, respectivamente, frente a explicaciones más relacionados con factores externos (Barberá, 1998).

A partir de aquí se postula la norma de la sociedad que no sólo describe lo usual sino que también prescribe lo que hay que hacer (Geis, 1993).

Los creadores de esta propuesta plantean que para modificar este proceso cíclico es necesario un cambio de roles y una fuerte acción re-educativa (Haslett, Geis y Carter, 1992).

La profecía autocumplida, aunque no aborda el proceso de autocategorización e identificación, explica el círculo de retroalimentación entre estereotipos y conducta en la interacción personal; y describe los mecanismos presentes en las interacciones entre individuos con unos roles estructurales determinados. Dicha interacción, como se verá en la perspectiva integradora de Deaux y Martín (2003), es un elemento en la construcción de la identidad de género.

\subsection{Modelos sociales}

Se van a etiquetar como tales a las Teorías del Aprendizaje Social y las Teorías de la Identidad Social de Género. 
a) Las Teorías del Aprendizaje Social (Bandura, 1977; Lott, 1994; Lott y Maluso, 1993; Mischel, 1973). De acuerdo con estas propuestas los procesos a través de los cuales se adquiere el género son los mismos que por los que se adquiere cualquier otra conducta: los de aprendizaje. Estas teorías han analizado el aprendizaje de conductas a partir de los modelos de referencia y del refuerzo recibido. Tres han sido los agentes socializadores principalmente estudiados: los cuentos, la televisión y las figuras progenitoras.

Los relatos ilustrados dirigidos a los más pequeños muestran datos nada tranquilizadores. Los análisis de contenido de los cuentos clásicos indican que los chicos son mostrados como "activos, dinámicos y con mayor valor que las chicas". Ellas son "pasivas, limpias, ordenadas, tranquilas, emotivas, soñadoras, amables y dóciles" (Turin, 1995, p. 7).

Los hombres son "responsables, creativos, heroicos, leales y capaces de la amistad y el desinterés". Las mujeres (salvo la madre que cuida y atiende o la princesa que se deja salvar y se casa con el príncipe) son "malévolas, frívolas, explotadoras, derrochadoras y sobre todo estúpidas" (Turin, 1995, p. 8).

Sin duda, la frase de una niña de siete años recogida por D'Hont y Vandewiele (1986) muestra el impacto que estos modelos pueden tener sobre los estereotipos, roles e identidades de los más pequeños: "Yo no puedo ser médico, solamente enfermera. Lo pone en mi libro".

Respecto a la televisión, la posibilidad de medir su influencia sobre una comunidad no televisiva vino de la mano de Williams, en 1986. Para ello evaluaron los roles de género antes de la implantación de este medio y lo compararon con una medida obtenida tras los dos primeros años de emisión. En ambos sexos se pudo observar la acentuación de los roles tipificados de género.

Otros tipos de diseños para evaluar el impacto de la televisión son los que correlacionan los informes de los progenitores sobre el tiempo de visionado televisivo de sus hijos y medidas de prejuicios de género. Pero esta técnica ha arrojado datos contradictorios (véase por ejemplo Zuckerman, Singer y Singer, 1980; Morgan, 1982).

En cuanto a las figuras progenitoras se ha hallado correlación entre la feminidad y la masculinidad de los padres con el estereotipo de los hijos (Repetti, 1984). Pero no todo los datos son esclarecedores (véase Maccoby y Jacklin, 1974, 1987).

Una posible explicación a estos resultados poco concluyentes puede ser que, tal y como plantea Doyle (1985), los modelos que los más pequeños utilizan como referencia no son sólo los de su grupo sexual. Parece que aquellos con los que tienen una fuerte vincula- ción afectiva y aquellos que son símbolo de poder y dominio también son figuras a imitar. En general, los datos tienden a reflejar que tanto chicos como chicas imiten más a los modelos masculinos (Slaby y Frey, 1975), una imitación que tal y como se explicará en la SIT (Tajfel, 1981 y Tajfel y Turner, 1986) puede ser debida a la búsqueda de una identidad social positiva, ya que éstos son los más valorados.

Las teorías del aprendizaje social tratan de explicar cómo, a través de un proceso educativo diferencial, se adquieren patrones conductuales de género. Pero no abordan los mecanismos intrapsíquicos responsables de la adquisición de las conductas ni como éstas influyen en el desarrollo del self.

b) Teorías de la Identidad Social de Género. Siguiendo la clasificación realizada por E. Barberá (1998) en este epígrafe se incluyen: la Teoría del Rol (Eagly, 1987), la Teoría de la Identidad Social y de la Autocategorización (Tajfel, 1981 y Tajfel y Turner, 1986) y la propuesta integradora de Deaux y Martin (2003). Todas ellas parten de la identificación grupal como eje de la identidad personal y por tanto de género.

b.1) Teoría del Rol Social de Género (Eagly, 1987). Una comunidad necesita estar organizada para garantizar los recursos económicos y los medios de subsistencia. Con esta finalidad se dividen las tareas y actividades responsables de la producción, división que necesita de normas que la garantice. Para ello se elabora un complejo sistema de reglas que asigna responsabilidades y roles a los miembros de la comunidad. Una vez realizada esta segmentación laboral se va a convertir en uno de los pilares básicos de la estructura social, a la vez que estable y regula las diversas relaciones intergrupales. Estas Relaciones también generan desigualdades sociales pues prescriben las diferencias, tanto en el ámbito público como en el privado.

Cada uno de los miembros de la comunidad va a adoptar un rol en esta estructura. De acuerdo con Mead (1934), mediante el rol los más jóvenes aprenden a asumir sus funciones y a entrar en la dinámica social en consonancia con los otros miembros. Las normas que rigen este proceso de interacción generan expectativas, pero ninguna sociedad posee un consenso total sobre las mismas. Por consiguiente, parece que los roles sociales son creados gracias a la interacción dinámica entre los ejecutores y el resto de la comunidad.

Los papeles sociales (Geis, 1993), junto con sus expectativas, forman parte de los valores culturales e influyen en el comportamiento de las personas. Por ello, 
a mayor solidez de la estructura social y mayor aceptación de dichas expectativas, más consistencia adquiere la ideología que mantiene el sistema. Se perpetúan así los roles y en consecuencia las desigualdades que éstos conlleven. La separación por roles, que adquiere carácter de norma, presenta un claro efecto sobre la identidad de género, ya que los roles definen la mayoría de las actividades de las personas, así como los mecanismos para participar en la sociedad (Escartí, Musitu y Gracia, 1988).

Los roles asignados en función del trabajo son el primer eslabón en la cadena de causalidad: de ellos se deriva la estereotipia y ambos determinan el autoconcepto de género. Cada puesto laboral lleva asociada una serie de demandas necesarias para su desempeño. Si un trabajo es habitualmente desarrollado por hombres se considera que el ser hombre implica esa serie de características: creencias que se plasman en diferencias reales a través de dinámicas de asignación y expectativas. Mediante la asignación se regulan las habilidades y motivaciones de hombres y mujeres orientándolas en la dirección de los estereotipos. Por otro lado, las expectativas generan el esquema de autocumplimiento reforzándolos.

Si se estudian las imágenes de género se puede observar cómo la tradicional concepción de mujer (más amable, cariñosa y comprensiva que el hombre) no se mantiene si se describe a una mujer trabajadora en lugar de a una ama de casa (Eagly y Steffen, 1984). Igualmente la ocupación de un puesto de alta responsabilidad en el mundo laboral les hace ser descritas tan independientes como sus compañeros varones, mientras que cuando poseen un trabajo de subordinadas se las considera más sumisas (Eagly y Wood, 1982). Mediante el mismo proceso a los hombres que desempeñan las tareas del hogar se les suponen tan sensibles como a las mujeres en las relaciones interpersonales (Eagly y Steffen, 1984), creencia que no se sostiene si es otra la ocupación.

En similar dirección señalan las investigaciones dirigidas a examinar las características que demanda un rol. Mientras a las ocupaciones de bajo estatus se les asignan los rasgos comunales: participantes, tendentes al contacto, a la apertura, a la unión y la cooperación (Bakan, 1966), a las posiciones más elevadas se les asocian los agentes (Bakan, 1966): iniciativa, control, tendencia a la actividad externa, autoprotección y autoexpansión (Conway, Pizzamiglio y Mount, 1996).

Esta teoría recoge muy bien la relación entre rol e identidad, pero su énfasis en el contenido específico de los roles laborales y los rasgos diferenciales de personalidad, agentes y comunales, ha suscitado propuestas alternativas (veáse Henley, 1977; Berger y Zelditch, 1985; Ridgeway, 1991; Deaux y Major, 1987). Además no explica las diferencias interindividuales de identidad entre personas que comparten roles y condiciona los cambios de identidad a los cambios de rol.

b.2) La Teoría de la Identidad Social y Teoría de la Autocategorización (Tajfel, 1981 y Tajfel y Turner, 1986).

Henri Tajfel explica cómo se construye la identidad social de las personas desde los mecanismos cognitivos y motivacionales presentes en las interacciones grupales. De acuerdo con este autor la identidad se define como "aquellos aspectos de la propia imagen del individuo que se derivan de las categorías sociales a las que percibe pertenecer" (Tajfel y Turner, 1986, p. 16), de tal forma que al definirnos como mujer u hombre estamos apelando a nuestra identidad social de género. La construcción de la misma supone un proceso de auto-estereotipaje por el que las actitudes, normas y conductas comunes al grupo de pertenencia pasan a formar parte de la identidad personal.

El primer proceso del que parte es la categorización. Las categorías sociales, además de facilitar el procesamiento de la información, llevan asociadas una valoración positiva o negativa. Es dicha valoración la responsable del nacimiento de la identidad social del sujeto. Tajfel, mediante el paradigma de grupo mínimo, halla que la mera y simple conciencia de pertenecer a un grupo conduce al favoritismo intragrupal y la discriminación intergrupal (Brown, 1985; Richardson y Cialdini, 1981; Tajfel, 1978, 1981, 1982; Turner, 1978, 1987;). La base de esta discriminación automática es la búsqueda de una identidad social positiva. El endogrupo sólo tiene valor si se percibe como superior al exogrupo, mediante la discriminación se facilita la diferenciación categórica y así se consigue la anhelada identidad social positiva.

Este doble efecto, favoritismo - discriminación, se vale del proceso de comparación social para la consecución y el mantenimiento de la identidad positiva (Turner y Oakes, 1986). Dicho proceso no requiere de un conflicto (Tajfel, 1982), sino que parece poseer un carácter espontáneo (véase Haeger, 1993).

Es la valencia de las categorías y la motivación por una identidad socialmente valorada lo que va a condicionar cuán identificados nos sentimos con nuestro grupo. Si en el proceso de comparación social nuestro grupo posee un bajo estatus pondremos en práctica estrategias para salvaguardar la identidad grupal y la autoestima. Estas estrategias van a depender de tres factores. El primero es la permeabilidad versus impermeabilidad entre los límites de los grupos. El sexo es una característica no permeable, lo que hace rígido e inflexi- 
ble el paso entre las fronteras grupales. El segundo factor es la estabilidad versus inestabilidad en las diferencias de estatus, que alude a la posibilidad de cambio. Por último, tenemos la legitimidad versus ilegitimidad, basado en la percepción de injusticia y arbitrariedad de la situación social (Tajfel y Turner, 1986). De acuerdo con este planteamiento las mujeres, grupo impermeable, inestable e ilegítimo, deben optar por la creatividad social, la redefinición de las características y/o la competición social. Han sido estrategias adoptadas por el movimiento feminista desde sus orígenes.

La teoría de H. Tajfel trata de explicar los cambios sociales derivados de los conflictos entre el individuo y la estructura social. Para ello recurre a los procesos psicológicos universales.

La Teoría de la Identidad Social (SIT) también ha sido la cuna de otras propuestas como ocurre con la Teoría de la Autocategorización (SCT)(Turner, 1985; Turner y otros, 1987). En ella se considera al autoconcepto un componente del sistema psicológico etiquetado como Yo. El Yo no lo forma un único autoconcepto, sino que hay distintos para las diversas situaciones, de tal forma que se activan unos $\mathrm{u}$ otros en función del contexto y momento concreto. Por tanto ante estímulos similares el sujeto se autocategoriza, es decir categoriza su Yo, como miembro o no del grupo. Dichas categorías poseen una estructura jerárquica con al menos tres niveles relevantes para el autoconcepto social, niveles que se relacionan entre sí. El más externo, que abarca a los siguientes, es el nivel supraordenado donde están las categorizaciones ligadas a la identidad humana frente a otras especies, en el segundo nivel se encuentran las categorías vinculadas a la diferenciación endogrupo - exogrupo y en el eslabón más subordinado residen las categorizaciones personales del Yo, las que recogen las diferencias interindividuales con los demás miembros del grupo. La categorización está condicionada por el proceso de comparación, al mismo tiempo que éste depende de aquélla. Luego, el contexto de referencia va a determinar la formación de las categorías. De acuerdo con Turner (1987) la autopercepción es parte de un continuo que varía desde la identidad personal hasta la identidad grupal.

Ambas teorías han recibido diversas críticas (para una mayor revisión véase Brown, 1995, 2000 y Turner, 1999), de las cuales se va a tratar una: su psicologicismo. La SIT, a pesar de considerar el contexto, pues tiene en cuenta la presencia de otros grupos, no explica bien ni presta suficiente atención al entorno social (Hogg y cols., 1995). No distingue entre las categorías sociales y el contexto inmediato de interacción social, mezclando todas las posibles interacciones sociales (Thoits y Virs- hup, 1997). También se le ha criticado que, al ser una teoría que trata de explicar los conflictos grupales, simplifica la feminidad y trivializa la relación entre hombres y mujeres (Skevington y Baker, 1989).

Por su parte la SCT considera el contexto inmediato, pero las características de éste dependen de la percepción del sujeto (Deaux y Martín, 2003) desplazando nuevamente el papel agente a los factores psicológicos frente a los sociales.

La Teoría de la Identidad Social es, sin lugar a dudas, una de las más elaboradas y completas de todas las expuestas. Su análisis grupal a partir de aspectos cognitivos y motivacionales le ha proporcionado unos principios amplios y flexibles. En palabras de Brown (2000), a pesar de las limitaciones que se le han observado, esta teoría representa un desafío para las nuevas investigaciones, puesto que posee los pilares adecuados sobre los que poder construir una explicación adecuada de tan complejo proceso.

b.3) Propuesta integradora de Deaux y Martin (2003). Ambas autoras hacen una revisión de dos de las disciplinas centradas en el estudio de la identidad: la sociología y la psicología. De cada una de ellas eligen la teoría más relevante en la explicación de la construcción de la identidad: la Teoría de la Identidad de Stryker (1980), en sociología, y la Teoría de la Identidad Social (Tajfel, 1981 y Tajfel y Turner, 1986), en Psicología. A partir de ambas plantean una solución integradora que finalice con el eterno debate sociedad versus individuo. Puesto que la SIT se ha abordado en el apartado anterior se va a esbozar brevemente la IT (Teoría de la Identidad) para así comprender mejor esta propuesta.

La Teoría de la Identidad (Stryker, 1980). Para la IT el proceso de identificación tiene lugar en la interacción entre los roles complementarios de una estructura específica (Stryker, 1980; Stryker y Serpe, 1982). De acuerdo con esta teoría la identidad es aquel conjunto de significados compartidos por un sistema. Dichos significados se interiorizan a través de los roles (Deaux y Martín, 2003). El origen de la identidad está en la estructura social y su función es mantener el orden establecido (Thoits y Virshup, 1997). Puesto que la estructura potencia las interacciones dentro de los límites definidos por ella e inhibe las demás, propicia el status quo de los roles (Stryker, 1997).

Sus autores distinguen tres niveles dentro de la estructura social. El nivel más general (large-scale) hace alusión a las variables sociodemográficas: edad, sexo, etnia y estatus económico. El nivel intermedio serían aquellas estructuras sociales organizadas como el colegio, el barrio, o el trabajo, en las que se adopta un rol 
concreto, rol que va a depender de la large scale. Es decir, si se toma la categoría sexo, mujeres y hombres van a adoptar roles distintos dentro del mismo ámbito laboral. Por último, el nivel próximo sería aquel contexto inmediato donde se producen las interacciones personales más cercanas. Son los roles adoptados en este nivel los que se interiorizan configurando la identidad. Dichos roles están determinados por las categorías sociodemográficas a las que se pertenece (Stryker, Serpe y Hunt, 2000). Según la IT el determinismo de variables físicas y sociales actúa por igual en todos los miembros del grupo.

Esta es una de las limitaciones que presenta la teoría sociológica. Tal y como muestran los estudios de la SIT, hay diferencias en la identificación de las personas con su grupo, pues quienes pertenecen a grupos de alto estatus se identifican más con el mismo que los de bajo estatus. Tampoco explica los procesos intraindividuales presentes en la identificación (Deaux y Martín, 2003) y cae casi en el inmovilismo de la identidad, ya que sus posibles cambios están sujetos a las modificaciones del rol (Hogg y cols. 1995).

Una propuesta integradora (Deaux y Martín, 2003). Esta propuesta quiere unir el análisis estructural de la IT, concretamente va a considerar el nivel más amplio de la estructura social (large scale) y el contexto inmediato de interacción, pero teniendo en cuenta los procesos cognitivos explicados por la SIT y la SCT en cada uno de estos niveles.

El primer elemento a explicar es la large scale o categorías. Al igual que lo define Stryke son aquellas categorías sociodemográficas que estructuran los grandes segmentos sociales. La identificación personal con estas categorías proporciona una red de interacción entre individuos que poseen los mismos significados. Además también facilita un contexto de comparación social donde, como indica la SIT, variables como el estatus pueden condicionar y motivar cambios en la identidad grupal.

El segundo elemento, la interacción interpersonal, alude a las interacciones que se producen cada día con las personas con las que compartimos una identidad categorial. En ellas se adoptan roles interdependientes que reproducen y refuerzan la identidad socialmente definida. Nuevamente en estas redes están presentes los mecanismos cognitivos explicados por la SIT. La identificación está condicionada por la búsqueda de una identidad social positiva, por ello si el grupo es de bajo estatus la persona pondrá en marcha diversos mecanismos para salvaguardar su autoestima e identidad grupal. Las mujeres, tal y como se expuso en la SIT, al ser el grupo de menor estatus tratan de redefinir sus características y competir socialmente.

Luego la identidad social es dinámica y se encuentra en un continuo proceso de negociación. Las interacciones grupales pueden reforzar o propiciar la identidad de un individuo dentro de su propio grupo. Frente a la estabilidad del nivel large scale, en la interacción interpersonal hay una continua reinterpretación de los significados de una identidad en un contexto concreto.

De acuerdo con este modelo el self es agente y producto a la vez. Los hombres y mujeres de acuerdo con la estructura social establecida son asignados a distintos grupos en base a su diferente apariencia sexual. Cada grupo lleva asociado una serie de pautas de sociales, comportamentales y psicológicas que aprenden. Por lo que se diría que el sí mismo es una adscripción colectiva. Pero las personas en sus interacciones guían su proceso de identificación por lo que es también una construcción subjetiva.

Este modelo es sin duda una interesante propuesta que supera las tendencias reduccionistas de la mayoría de las teorías pero como recogen sus autoras aún necesita apoyo empírico (Deaux y Martín, 2003).

\section{CONCLUSIÓN}

De acuerdo con el objetivo del presente artículo se han revisado las limitaciones y aportaciones de diversas teorías sobre la construcción del self de género. Inicialmente se han abordado los modelos cognitivos cuya principal aportación ha sido destacar el papel de la categorización. Sin duda, si no diferenciamos entre distintos grupos sociales, no tendremos la necesidad de identificarnos con unos u otros.

La profecía autocumplida explica otro de los elementos del puzzle: los estereotipos. Describe cómo actúan en las interacciones personales y en la construcción de la realidad: cómo se refuerzan las creencias dominantes de un grupo social y cómo provocan situaciones que las mantienen.

Desde el aprendizaje social se analizan los modelos de referencia, así como la adquisición y manifestación de conductas tipificadas.

La teoría del rol informa de cómo los roles, dentro de una determinada estructura social productiva, condicionan las relaciones intergrupales, así como el vínculo entre éstos y los estereotipos.

Todos estos elementos: la categorización, los estereotipos, los roles, los modelos y el aprendizaje de conductas son partícipes en la construcción del self, pero por si 
solos no pueden explicarla. De ahí que estos modelos explicativos sean parciales o reduccionistas.

Van a ser dos teorías psicosociales, la SIT y la propuesta de Deaux y Martin (2003), las que traten de dar una respuesta multicausal al fenómeno del self de género. Para ello van a organizar y agrupar estas aportaciones además de nuevos elementos.

La gran contribución de Tajfel y Turner es incluir el elemento motivacional dentro de una perspectiva intergrupal. Además le otorgan un papel central a la categorización y a los estereotipos. Pero la SIT no trata en profundidad el contexto ni los roles estructurales que proporciona una comunidad.

Esta limitación es la que trata de solventar la propuesta de Deaux y Martin. Su fusión de los procesos psicológicos descritos por la SIT con los elementos estructurales y contextuales analizados por la ST dan una visión integral a cómo se organizan e interrelacionan los procesos responsables de la construcción, modificación y mantenimiento del self de género.

Estas autoras consiguen analizar los mecanismos presentes en el selfcomo fenómeno psicosocial, poniendo el acento en ambas disciplinas. Tal y como ellas afirman, quedan cuestiones por perfilar en el modelo: la motivación y las funciones de la identidad social en los diversos niveles contextuales o la concepción de las redes sociales frente a las dinámicas intra-intergrupales (Deux y Martin, 2003). Por ello, las investigaciones futuras deben ir orientadas a clarificar y matizar estos elementos teóricos y a aportar un cuerpo empírico a este modelo integrador.

\section{REFERENCIAS}

Bakan, D. (1966). The duality of human existence. Chicago: Rand McNally.

Bandura, A. (1977). Social learning theory. Englewood Cliffs, New Jersey: Prentice-Hall

Barberá E. (1998). Psicología del género. Barcelona: Ariel Psicología.

Bem, S. (1974). The measurement of psychological androgyny. Journal of Consulting and Clinical Psycho$\log y, 42,155-162$.

-,(1981). The BSRI and gender schema theory: a reply to Spence and Helmreich. Psychological Review, 88, $369-371$.

Berger, J. y Zelditch, M. Jr. (1985). Status, rewards and influence: How expectations organize behavior. San Francisco: Jossey -Bass.

Biernat, M., Manis, M. y Nelson, T. (1991). Stereotypes and standards of judgment. Journal of Personality and
Social Psychology, 60, 485-499.

Bonilla, A. (1998). Los roles de género. En J. Fernández (coord.), Género y sociedad. Madrid: Pirámide.

Brown, R. (1995). Prejuicio, su psicología social. Madrid: Alianza Editorial.

-,(2000). Social Identity: past achievements, current problems and future challenges. European Journal of Social Psychology, 30, 745-778.

Carver, P.R., Yunger, J.L. y Perry, D. G. (2003). Gender identity and adjustment in middle childhood. Sex Roles, Vol 49, No 3/4

Conway, M., Pizzamiglio, M. T. y Mount, L. (1996). Status, communality and agency: implications for stereotypes of gender and other groups. Journal of Personality and Social Psychology, 71, 25 - 38.

D’Hont, W. y Vandewiele, M. (1986). Les écoliers nigériens de 7 a 12 ans et le test de l'arbre. Cahiers de Sociologie Economique et Culturelle, Dec, No 6, $41-80$.

Darley, J. M. y Fazio, R. H. (1980). Expectancy confirmation processes arising in the social interaction sequence. American Psychologist, 35, 867 - 881.

Deaux, K. y Major, B. (1987). Putting gender into context: an interactive model of gender - related behavior. Psychological Review, 94, 369 - 389.

-, y Martin, D. (2003). Interpersonal networks and social categories: Specifying levels of context in identity processes. Social Psychology Quarterly, Vol, 66, No2, 101-117.

Doyle, A. (1985). Sex and gender: the human experience. Dubuque Iowa: Wm., C. Brown.

Eagly, A. H. (1987). Sex differences in social behavior: a social interpretation. Hillsdale, NJ: LEA.

-,y Steffen, V. J. (1984). Gender stereotypes stem from the distribution of women and men into social roles. Journal of Personality And Social Psychology, 46, 735-754.

-,y Wood, W. (1982). Inferred sex differences in status as a determinant of gender stereotypes about social influence. Journal Of Personality And Social Psycho$\log y, 43,915-928$.

Escartí, A. Musitu, G. y Gracia, M. D. (1988). Estereotipos de género y roles sexuales. En J. Fernández (coord.), Nuevas perspectivas en el desarrollo del sexo y el género. Madrid: Pirámide.

Geis, F. L. (1993). Self - fulfilling prophecies: a social psychological view of gender. En A. E. Beall y R. J. Sternberg (Eds.), The psychological of gender. Nueva York: Guilford Press.

Golombok, S. y Fivush, R. (1994). Gender development. Cambridge: Cambridge University Press.

Haeger, G. (1993). Social and temporal comparisons in
REVISIÓN 
an european context. Sin publicar. Msc. Thesis: University of Kent.

Hare-Mustin, R.T. y Marecek, J. (1994). Los sexos y el significado de la diferencia: postmodernidad y psicología. En R. T. Hare-Mustin y J. Marecek (Eds.) Marcar la diferencia. Psicología y construcción de los sexos. Barcelona: Herder.

REVISIÓN

Haslett, B., Geis, F.L. y Carter, M. (1992). The organizational woman: Power and paradox. Nueva York: Ablex.

Henley, N. M. (1977). Body politics: power, sex and nonverbal communication. Englewood Cliffs, Nj: Prentice - Hall.

Hogg, M.A., Terry, D.J. y White, K.M. (1995). A tale of two theories: Critical comparison of Identity Theory and Social Identity Theory. Social Psychology Quarterly, Vol 58, 255-269.

Huston, A.C. (1983). Sex Typing. En P. H. Mussen (Eds.) Handbook of child psychology. Socialization, personality and social development, vol $I V$. Nueva York: Wiley.

Koestner, R y Aube, J. (1995). A multifactorial approach to the study of gender characteristics. Journal of Personality, 63, 3, 681-701.

Kohlberg, L. (1966). A cognitive developmental analysis of children's sex role concepts and attitudes. En E. E. Maccoby (Eds.), The development of sex differences. Stanford: Stanford University Press.

López, F. (1988a). Adquisición y desarrollo de la identidad sexual y de género En J. Fernández (coor). Nuevas perspectivas en el desarrollo del sexo y del género. Madrid: Pirámide.

-,(1988b). Identidad sexual y de género en la vida adulta y vejez. En J. Fernández (coor). Nuevas perspectivas en el desarrollo del sexo y del género. Madrid: Pirámide.

Lott, B. (1994). Naturalezas duales o conducta aprendida: el desafío de la psicología feminista. En R. T. Hare-Mustim y J. Marecek (Eds.) Marcar la diferencia. Psicología y construcción de los sexos. Barcelona: Herder.

-,y Maluso, D. (1993). The social learning of gender. En A. E. Beall y R. J. Sternberg (Eds.) The psychology of gender. Nueva York: Guilford Press.

Maccoby, E. E. y Jacklin, C. N. (1974). The psychology of sex differences. Stanford: Stanford University Press.

-,(1987). Gender segregation in chilhood. Advances in child development and behaviour, 20, 239 - 287.

Markus, H., Crane, M., Berstein, S. y Siladi, M. (1982). Self-schemas and gender. Journal of Personality and Social Psychology, 42, 38-50.

Martin, H. y Halverson, C. F. (1983). Gender constancy: A methodological and theoretical analysis. Sex
Roles, 9, 775-790.

Mead, G. H. (1934). Mind, self and society. Chicago: University of Chicago Press.

Merton, R.K. (1948). The self-fulfilling prophecy. Antioch Review, 8, 193-210.

Mischel, W. (1973). Toward a cognitive social learning reconceptualization of personality. Psychological Bulletin, 80 (4), 252-253.

Morgan, M. (1982). Television and adolescents' sex role stereotypes: a longitudinal study. Journal of Personality and Social Psychology, 64, 198 - 210.

Piaget, J. (1966). The moral judgement of the child. Nueva York: Free Press.

Repetti, R. L. (1984). Determinants of children's sex stereotyping: parental sex - role traits and television viewing. Personality and Social Psychology Bulletin, 10, 457 - 468.

Richardson, K. D. y Cialdini, R. B. (1981). Two indirect of image management: basking and blasting, Journal of Personality and Social Psychology Vol 39 (3), $406-415$.

Ridgeway, C. L. (1991). Gender, interaction and inequality. Nueva York: Springer.

Rosenthal, R. y Jacobson, L. (1968). Pygmalion in the Classroom: teacher expectations and student intellectual development. Nueva York: Holt, Rinehart y Winston.

Silka, L.J. y Maslach, C. (1987). Gender roles and the categorization of gender relevant behavior. Sex Roles, 22, 133-150.

Skevington, S. y Baker, D. (1989). Introduction. En S. Skevington y D. Baker (Eds.) The social identity of women. London: Sage.

Slaby, R. G. y Frey, K. S. (1975). Development of gender constancy and selective attention to same sex models. Child Development, 46, 849 - 856.

Snyder, M. (1981). On the self - perpetuating nature of social stereotypes. En D. L. Hamilton (Eds.), Cognitive Processes in Stereotyping and Intergroup Behaviour. Nueva York: Lawrence Erlbaum.

Spence, J.T. (1993). Gender-related traits and gender ideology: evidence for a multifactorial theory. Journal of Personality and Social Psychology, 64 (4), 624-635.

-, Helmreich, R.L. y Stapp, J. (1974). The personal attributes questionnaire: a measure of sex role stereotypes and masculinity-feminity. ISAS Catalog of Selected Documents in Psychology, 4, 43.

Stryker, S. (1980). Symbolic Interactionism: a social structural version. Menlo Park, CA: Cummings.

-,(1997). In the beginning there is society: Lessons from a Sociological Social Psychology. En C. McGarty y A. Haslam (Eds.) The message of Social Psychology. London: Blackwell 
-, y Serpe, R. T. (1982). Commitment, identity salience and role behavior: Theory and research example. En W. Ickes y E. S. Knowles (Eds.) Personality, roles and social behaviour. New York: Springer.

-, Serpe, R. T. y Hunt, M.O. (2000).Social structure and commitment: A study of blacks, latinos and whites. Unpublished manuscript.

Tajfel, H. (1978). Differentiation between Social Groups: Studies in the social psychology of intergroup relations. Londres: Academic Press.

-,(1981). Social identity and intergroup relations. London: Cambrige Univ.Press.

-,(1982). Social psychology of intergroup relations. Annual Review of Psychology, 33, 1-30.

-,y Turner, J. C. (1986). The social identity theory of intergroup behaviour, pp.7-24; en Worchel, S. y W. G. Austin (Eds.), Psychology of Intergroup Relations. Chicago: Nelson.

Thoits, P. A y Virshup, L.K.(1997). Me's and we's: Forms and functions of Social Identities. En R. D. Ashmore y L. Jussim (Eds.) Self and Identity: fundamental issues. New York: Oxford University Press.

Turin, A. (1995). Los cuentos siguen contando: algunos reflexiones sobre los estereotipos. Madrid: Horas y Horas.

Turner, J. C (1978). Social comparison, similarity and ingroup favouritism. En H. Tajfel (ed.), Differentiation between Social Groups: Studies in the social psychology of intergroup relations. Londres: Academic Press.

-, (1985). Some Categorization and the Self-Concept: A social Cognitive Theory of Group Behavior. E. J. Lawler (ed.), Advances in Group Processes, Vol. 2, (pp. 77-122). Greenwich, CONN: Jai Press.

-,(1987). Rediscovering the social group: a self-categorization theory. Oxford: Basil Blackwell.

-,(1999). Some current issues in research on social identity and self categorization theories. En Social Identity, Ellemers, N., Spears, R., Doosje, B. (Eds.), Blackwell: Oxford, 6-34.

-, Hogg, M. A., Oakes, P.J., Reicher, S.D., Wetherell, M. S. (1987). Rediscovering the social group: a self-categorization theory. Blackwell: Oxford.

-, y Oakes, P. J. (1986). The significance of the social identity concept for social psychology with reference to individualism, interactionism and social influence. British Journal of Social Psychology, Vol 25 (3), $237-252$.

Zuckerman, D.M., Singer, D. G. y Singer, J. L. (1980). Children's television viewing, racial and sex - role attitudes. Journal of Applied Social Psychology, 10, $281-294$. 